\title{
La construction des institutions municipales au Burkina Faso : enjeux, contraintes et potentialités
}

\section{Pamphile Sebahara}

\author{
(2) OpenEdition \\ Journals \\ Édition électronique \\ URL : http://journals.openedition.org/apad/4126 \\ DOI : $10.4000 /$ apad. 4126 \\ ISSN : 1950-6929 \\ Éditeur \\ LIT Verlag \\ Édition imprimée \\ Date de publication : 1 janvier 2012 \\ Référence électronique \\ Pamphile Sebahara, "La construction des institutions municipales au Burkina Faso : enjeux, \\ contraintes et potentialités », Bulletin de l'APAD [En ligne], 34-36 | 2012, mis en ligne le 19 juillet 2013, \\ consulté le 10 décembre 2020. URL : http://journals.openedition.org/apad/4126 ; DOI : https://doi.org/ \\ 10.4000/apad.4126
}

Ce document a été généré automatiquement le 10 décembre 2020.

Bulletin de l'APAD 


\title{
La construction des institutions municipales au Burkina Faso : enjeux, contraintes et potentialités
}

\author{
Pamphile Sebahara
}

\section{Introduction}

1 La présente communication se propose d'apporter une contribution à l'objectif du colloque, à savoir "engager l'anthropologie pour le développement et le changement social». Une réflexion sur le processus de construction des institutions municipales au Burkina Faso me semble nécessaire pour au moins deux raisons. D'une part, en matière de développement des sociétés africaines, l'enjeu le plus important ne réside pas dans le nombre d'infrastructures construites ou de services offerts à la population à un moment donné, mais plutôt dans la mise en place et le renforcement des institutions capables d'assurer la régulation des rapports sociaux et de garantir l'intérêt général à travers la production des normes, le contrôle de leur respect et la sanction des transgressions. D'autre part, si plusieurs recherches en anthropologie voire en science politique ont été réalisées sur certains aspects des politiques de décentralisation, l'analyse des processus de construction des institutions publiques - ou voulues comme telles - et de production de règles collectives qui régissent le vivre ensemble en milieu urbain ne semble pas avoir fait l'objet de beaucoup d'intérêt, du moins à ce stade. Or, le développement conçu comme un processus d'amélioration des conditions de vie des gens ne serait possible sans des institutions capables d'assurer la régulation sociale et politique, y compris dans divers conflits entre les acteurs. C'est pourquoi je propose une contribution à travers l'esquisse d'une socio-anthropologie de la construction des institutions publiques ou du moins étatiques car le concept de « public » est lui-même problématique. 
2 L'analyse est basée sur des données collectées pendant un séjour de plus de 3 ans au Burkina Faso (entre 1999 et 2003) en tant qu'assistant technique auprès de la Commission nationale de la décentralisation (CND) chargé d'une recherche-action sur la mise en place des communes. Les observations et les informations présentées ici concernent principalement la commune de Ziniaré, située dans la région du Plateau central en pays mossi. Si nous avons choisi de centrer notre analyse sur Ziniaré, c'est parce que les caractéristiques de cette commune en voie d'urbanisation rapide se retrouvent également dans d'autres communes dans lesquelles j'ai menées les enquêtes à la même période, en l'occurrence Banfora, Boromo, Pouytenga, Ouahigouya et Koudougou. Chaque localité a néanmoins ses spécificités qui pourraient être traitées dans une étude comparative qui n'est pas l'objet de notre propos.

3 Trois questions relatives à la construction des institutions municipales constituent le fil conducteur de la présente analyse :

4 - L'origine des décisions des autorités nationales d'adopter la politique de décentralisation et la perception que les différentes catégories de la population en ont eue.

5 -Les modalités de gestion des affaires administratives (état civil) et des politiques municipales en matière d'assainissement, y compris la gestion des déchets. L'hypothèse de base est que la légitimité des nouvelles institutions aux yeux des populations sera fonction des capacités des premières à mener des politiques qui répondent à leurs besoins.

6 - Les modalités de collaboration ou non entre le conseil municipal et les autres acteurs institutionnels présents dans la commune, notamment les organisations de la société civile et les projets de développement.

7 Cette tentative d'expliciter les multiples dynamiques institutionnelles à l'œuvre pourrait contribuer à une meilleure compréhension des conditions favorables au développement des institutions légitimes, capables de garantir l'intérêt général, c'est-à-dire assurer la sécurité et gérer les services à la population tout en régulant les multiples interventions qui y ont lieu. Il me semble que c'est un des rôles qu'une socio-anthropologie ouverte aux autres sciences sociales pourrait jouer.

\section{La décentralisation au Burkina Faso : état des lieux}

Depuis le début des années 90, plusieurs pays d'Afrique de l'Ouest ont adopté des politiques de décentralisation de l'Etat. Celles-ci consistent en la création de collectivités territoriales avec une certaine autonomie administrative, financière et politique. Ces institutions locales sont mises en place dans un environnement où coexistent d'autres institutions, étatiques, associatives, coutumières, religieuses et les projets de développement qui sont tout aussi légitimes, parce qu'elles remplissent diverses missions au profit des populations de ces localités. Au Burkina Faso, la décentralisation a été lancée à l'occasion de l'adoption de la Constitution de 1991 avec l'objectif affiché de promouvoir la démocratisation et le développement local. Elle a été mise en œuvre d'abord en milieu urbain en 1995, ensuite en milieu rural en 2006. 


\section{Une décentralisation par le haut}

La politique de décentralisation de l'Etat en cours au Burkina Faso a une double origine : la Constitution du 2 juin 1991 qui rétablit les institutions démocratiques et instaure la quatrième République d'une part, et le Plan d'Ajustement Structurel (PAS) qui, signé la même année avec la Banque Mondiale, prévoit la décentralisation parmi ses mesures d'accompagnement ${ }^{1}$.

10 La Constitution de 1991 affirme le principe de l'organisation du territoire en collectivités territoriales dans les articles 143, 144 et 145. En mai 1993, cinq lois de décentralisation ont été adoptées et ont permis l'organisation des premières élections dans trente trois communes urbaines en 1995. Elles garantissent l'autogestion des collectivités territoriales en prévoyant l'élection des membres des organes délibérants et en fixent les règles de fonctionnement. La même année (1993), une Commission Nationale de Décentralisation (CND) a été créée comme structure chargée de proposer un avant-projet de textes d'orientation de la décentralisation et de piloter le processus sous la tutelle du Premier Ministère.

11 En 1998, les Textes d'orientation de la décentralisation (TOD), composés de quatre lois cadres, précisent les lois de 1993 et portent sur l'orientation de la décentralisation, l'organisation de l'administration du territoire, l'organisation et le fonctionnement des collectivités locales et la programmation de la mise en œuvre de la décentralisation. Ils ont été modifiés par la loi n013-2001/AN du 2 juillet 2001 portant création de 13 régions qui sont à la fois collectivités locales et circonscriptions administratives. ${ }^{2}$

12 Le 21 décembre 2004, le Code général des collectivités territoriales a été adopté, avec l'option d'une communalisation intégrale. Il détermine et définit les deux types de collectivités territoriales; à savoir une collectivité de base (commune urbaine et rurale) et une collectivité de coordination (la région); et organise l'agencement général des cadres de gestion déconcentrée et décentralisée. Il remplace les lois antérieures et constitue la base juridique qui a permis l'organisation des élections municipales dans les communes urbaines et rurales en 2006. Dans ce contexte, le pays est organisé en collectivités territoriales résultant de la décentralisation, et en circonscriptions administratives qui relèvent de la déconcentration.

13 Il convient de garder en mémoire que nos enquêtes ont eu lieu pendant la période d'application des Textes d'orientation de la Décentralisation (TOD), soit entre 1998 et 2005. Selon ces derniers, « les collectivités locales concourent avec l'Etat à l'administration et à l'aménagement du territoire, au développement économique, social, éducatif, sanitaire, culturel et scientifique ainsi qu'à la protection, à la mise en valeur des ressources naturelles et à l'amélioration du cadre de vie $»^{3}$ A l'époque, trois principes directeurs devaient guider la décentralisation: la progressivité dans la mise en œuvre de la réforme institutionnelle (loi n⿳040/98/AN, art.14), la subsidiarité dans la répartition des compétences entre l'Etat et les collectivités locales (loi nº 040/98/AN, art.15), et le transfert concomitant aux collectivités locales des moyens et des ressources nécessaires à l'exercice normal de ces compétences dans le cadre des transferts de compétences de l'Etat aux collectivités locales (loi n 040/98/AN, art.16). La décentralisation devait en outre être accompagnée par une déconcentration 
adéquate des services de l'Etat dans le but de renforcer les capacités d'action des collectivités locales (loi n 040/98/AN, art. 3).

$14 \mathrm{Si}$ l'approche progressive a permis à la Commission nationale de la décentralisation (CND) de mener des campagnes de sensibilisation et de dialogue avec les populations à travers le pays, elle a eu aussi pour résultat le maintien du monde rural en dehors de la décentralisation pendant dix ans. L'espace rural est resté soumis à des régimes de gouvernementalité marqués par le pluralisme et une tradition de participation sans droit d'opposition ${ }^{4}$. Il est difficile d'évaluer l'impact de ces actions de mobilisation de la population en termes d'appropriation de la réforme. Ce que montrent nos enquêtes, c'est une certaine indifférence des catégories pauvres de la population vis-à-vis de cette réforme institutionnelle. Si ces populations ont déjà entendu parler de la décentralisation, elles ajoutent rapidement que c'est une décision politique de l'Etat central (« de Ouagadougou ») comme toutes les réformes qui ont déjà eu lieu auparavant. A ce propos, il est important de noter que le Burkina Faso avait déjà connu d'autres tentatives de décentralisation avant 1991. Celles-ci avaient été traduites par une série de lois cadres et peuvent être groupées en trois périodes : la loi du 2 février 1960 ; la tentative de décentralisation de 1974 à 1981; les tentatives de réforme institutionnelle de 1983 à 1989. Autrement dit, la décentralisation n'est pas un fait nouveau dans le pays.

15 L'examen des expériences passées de la décentralisation témoigne, d'une part, d'une volonté affichée dans les textes de loi de réorganiser le territoire, et, d'autre part, des difficultés à mettre ces principes juridiques et réglementaires en pratique. En effet, ces tentatives de décentralisation et de déconcentration d'avant 1991 n'ont pas abouti à une mise en œuvre effective, soit en raison d'un changement de régime gouvernemental, soit en raison d'un enlisement du processus, soit par faute de moyens humains et financiers, ou en raison des trois facteurs à la fois. Il a fallu également près de 15 ans pour que le processus lancé en 1991 et surtout en 1995 avec les premières élections dans les communes urbaines soit étendu sur l'ensemble du pays. Cela illustre le caractère politique de la décentralisation contrairement à certaines approches des donateurs qui tendent à mettre l'accent sur les aspects techniques de la réforme.

\section{Regards d'en bas : une indifférence face à la réforme de l'Etat}

Face à une décentralisation impulsée d'en haut, j'ai cherché à saisir la perception que les acteurs sociaux vivant dans la ville de Ziniaré ont de cette réforme institutionnelle à travers des discussions autour de questions relatives à la façon dont ils perçoivent la création des collectivités locales. La commune de Ziniaré où ont eu lieu les entretiens, est le chef-lieu de la Région du Plateau Central. Elle résulte du rapprochement de deux villages historiquement déterminants dans l'histoire de la chefferie mossi, à savoir Ziniaré et Guiloungou. Son marché qui a lieu tous les trois jours est réputé pour avoir été le premier centre d'échanges du plateau mossi. La ville connaît beaucoup de mutations depuis 1995, date à laquelle la localité a été érigée en commune de plein exercice dans le cadre de la mise en œuvre de la décentralisation. Plusieurs facteurs contribuent à son urbanisation croissante: l'arrivée de l'électricité, du téléphone et d'une route goudronnée au 
début des années 1990 ; l'ouverture de nombreuses petites 'entreprises' privées ; un plan cadastral couvrant le territoire communal et l'attribution de plus de 7000 parcelles par la mairie ; les cultures maraîchères autour du barrage de Tamissi et le fait qu'elle est la commune d'origine et de résidence du Chef de l'Etat Blaise Compaoré. Par ailleurs la présence de plus en plus grande des services administratifs, qu'ils soient municipaux, départementaux, provinciaux, régionaux, des organisations non gouvernementales (ONG) et associations de développement, tend à amplifier les transformations de la localité. Ainsi son statut de carrefour administratif, de part son impact sur l'évolution démographique et sur les dynamiques socio-économiques et culturelles, constitue un facteur clef de l'urbanisation.

17 La création de la commune inaugure la mise en place d'un cadre institutionnel relativement autonome pour la gestion des affaires locales. Pourtant, cet événement a priori important, notamment l'élection du premier conseil municipal le 12 février 1995, ne semble pas avoir marqué de façon particulière ses habitants rencontrés entre 1999 et 2003. Nous avons cherché à savoir comment la population s'y est impliquée et dans quelle mesure elle était informée de ce qui se passait ${ }^{5}$. Un responsable d'une ONG souligne à propos des différents changements administratifs de la commune: "Ce sont des décisions qui viennent de Ouagadougou. Ça n'intéresse pas tellement les gens. Ils savent qu'ils doivent faire les papiers et ils s'en vont au commissariat pour cela ou à la mairie. En réalité, les gens ne s'intéressent pas tellement à la politique. Ils savent qu'ils doivent aller voter. C'est leur marché qui les intéresse ou leurs problèmes. » Un membre de l'association de l'association des commerçants et des artisans de la place partage ce point de vue : «Pour la création de la commune, c'est une décision politique qui a créé les communes de plein exercice. La commune de Ziniaré fait partie des trente trois communes de plein exercice, qu'ils ont créées en 1995. » Ce dernier reconnaît toutefois que les membres de leur association peuvent participer «à titre individuel » aux partis politiques. Un responsable religieux confirme l'idée que la création de la commune résulte de la décision du gouvernement central. Pour lui par contre, les organisations religieuses, et notamment leurs responsables, peuvent diffuser les informations à la population et donner le bon exemple pour mettre en œuvre certaines actions de la mairie: "la création de la commune est surtout l'œuvre de la révolution. Mais c'est la décentralisation qui l'a renforcée. Quand il y a des élections ils nous demandent d'informer parce que nous avons le sens de l'information. Si nous trouvons que c'est juste, nous le faisons. Je participe aussi aux votes parce qu'il y a des gens qui sont réticents. Il faut savoir montrer aux gens que la politique n'est pas le côté négatif qu'ils en ont. Quand il y a des réunions ils m'informent et j'envoie des gens parce que cela tombe très souvent au moment où je suis occupé.»

18 Il semble dès lors que la population n'ait pas saisi dès le début l'importance du processus de décentralisation lancé par l'Etat avec l'appui des donateurs alors qu'il visait, officiellement du moins, la promotion du développement local et de la démocratisation à travers la mise en place des autorités proches de la population. Ce décalage entre les intentions des décideurs politiques nationaux et acteurs sociaux constituent un des éléments à approfondir si l'on veut contribuer 
effectivement au renforcement des institutions susceptibles de garantir l'intérêt général.

\section{Les institutions communales en action}

Les nouvelles institutions communales se sont mises en place dans un contexte de "dénuement " sur le plan matériel, humain et financier à l'exception des grandes agglomérations où il y avait déjà des agents publics. En effet, les grandes aglomérations disposaient déjà, avant 1991, d'un statut de commune même si elles étaient dirigées par des fonctionnaires nommés par le Gouvernement, c'est-à-dire non élus par la population. Elles avaient des agents publics ainsi que du matériel humain et financier. Cependant, les petites communes nouvellement créées, à l'instar de Ziniaré, ont essayé de répondre aux attentes de la population par la mise en place entre autres, d'infrastructures scolaires, sanitaires, routières ou d'assainissement Ce sont ces actions, réalisées par les équipes municipales issues des élections de 1995 à travers le pays, qui explique le début du changement de perceptions de certaines catégories de la population quand je leur ai posé la question de savoir s'ils pouvaient identifier des exemples d'effets concrets qui seraient liés à l'avènement de la mairie.

\section{Perceptions des effets et impacts de l'avènement de la mairie}

Selon le premier maire de la commune (1995-2000), l'avènement de la mairie est une bonne chose parce qu'elle permet d'adopter une organisation sociopolitique moderne. Il souligne toutefois la nécessité de ne pas copier le modèle européen parce que les contextes sont différents : "Si nous sommes en démocratie, il est normal que la population élise ses gouvernants. Il faut que la majorité prime sur la minorité. Le système démocratique est le bienvenu, nous l'avons adopté dans nos actions jusqu'à ce jour. Notons que l'Afrique et l'Europe ont chacune leurs spécificités. Pourtant nous avons tendance à tout copier de l'Europe ». Le maire souligne en particulier les contraintes que rencontrent les élus municipaux dans l'exercice de leurs activités à cause, entre autres, du statut bénévole de leurs fonctions.

21 Selon les élus municipaux interviewés, la création de la mairie favorise la participation de la population aux actions de développement de leur localité. Cette participation se fait sous plusieurs formes : la contribution financière à certains projets comme le lotissement, le payement des taxes, la main-d'œuvre dans la construction d'écoles et/ou dans l'aménagement de routes communales. Selon le premier maire de la commune en 1995/2000, «faire participer la population aux actions entreprises par la mairie est la seule garantie de succès parce que cela permet de trouver un équilibre entre les pratiques coutumières en vigueur et les nouvelles règles de l'administration publique». Cependant, les difficultés ne manquent pas dans la gestion quotidienne des affaires communales.

$22 \mathrm{Au}$ cours du premier mandat du conseil municipal (1995-2000), la population a contribué financièrement à la mise en œuvre du projet de lotissement à travers le payement des taxes par ceux qui voulaient acquérir une parcelle. Selon le maire : "L'Etat n'investit plus dans le lotissement. Il fallait donc trouver des initiatives qui 
impliquent la population dans la collecte des fonds pour l'entreprise qui allait faire le travail parce que les lotissements coûtent chers. Nous avons demandé à chaque citoyen qui voulait une parcelle de participer avec une somme de 20000 FCFA (pour les ressortissants de la commune, les autochtones), de 30000 FCFA (pour les ressortissants de la province), et de 50000 FCFA (pour tous les autres). Cela a permis de faire le lotissement. Voilà une forme de participation très importante qui mérite une attention parce que la décentralisation a responsabilisé tout un chacun. Si au départ (1995) nous avions un budget de 11 millions FCFA et qu'aujourd'hui, en 2000 , nous sommes à 61 millions FCFA, cela veut dire que chacun a pris conscience du fait qu'il doit payer ses taxes pour ses marchandises, ses armes, ses charrettes, ses kiosques afin de construire sa commune. L'évolution du budget est un signe que la décentralisation est une bonne chose. Les gens apprennent qu'il ne faut pas toujours tendre la main au niveau de l'Etat». Néanmoins, si on se réfère aux témoignages recueillis, la participation dans la prise de décisions, y compris le choix des actions prioritaires, ne semble pas être reconnue comme une forme de participation de la population à la vie communale.

23 Les cadres des services déconcentrés et des projets de développement basés dans la commune reconnaissent également les avantages liés à l'avènement de la mairie : la proximité de l'administration pour les habitants, la participation de la population aux actions initiées par la mairie à travers le payement des taxes, la réalisation des travaux d'intérêt général et les contributions financières pour la mise en œuvre du lotissement. Ils expriment toutefois des soucis par rapport à l'impact du statut bénévole des élus municipaux sur le fonctionnement de la mairie.

24 Un médecin du Centre médical pense que le «bénévolat constitue un frein au fonctionnement efficace des services municipaux. Il est nécessaire de trouver une solution à la rémunération des élus municipaux parce que les gens sont réticents à travailler bénévolement ». Par ailleurs, les agents des services déconcentrés et des projets de développement insistent sur l'importance, pour la mairie, de «s'attaquer aux problèmes concrets que les gens rencontrent quotidiennement». Ils citent entre autres, "les problèmes d'eau courante, de l'assainissement, de l'aménagement des routes, des dépôts d'ordures ménagères par ici et par là dans la ville, etc. ». Selon un chef de projet, " la commune ne peut pas relever toute seule les défis de son développement, parce que certains problèmes dépassent les moyens dont elle dispose».

25 Le point de vue des composantes de la société civile (ONG et associations locales, organisations religieuses, syndicales, coopératives) rend compte d'une conception paradoxale de leur rôle dans la gestion des affaires locales et par là dans le développement communal. C'est ce que nous avons appelé le «malentendu de la société civile $»^{6}$.

26 Pour ces acteurs, la politique de la décentralisation et la création des communes qui va de pair, est perçue comme une réforme de l'Etat parmi d'autres que le pays avait déjà connues telles que la création des départements ou des provinces. L'initiative vient de l'Etat et c'est lui qui la met en œuvre. Ces composantes de la société civile reconnaissent toutefois les avantages de la présence de la mairie: facilité et rapidité des démarches administratives, disponibilité des agents municipaux et des élus, ainsi que la réalisation de plusieurs projets de 
développement. Selon plusieurs membres de la société civile interviewés, "la création de la commune et sa gestion relèvent du champ politique alors qu'ils sont apolitiques de par leur statut associatif». Selon un responsable d'une ONG locale : "Nous sommes régis par une loi qui stipule que les Associations sont apolitiques alors que la mise en place des structures au niveau communal suppose d'être intégré d'une manière ou d'une autre dans l'administration ou avoir des aspirations politiques. Il y a des partis (politiques) au niveau national qui ont des ramifications sur le territoire. Ce sont ces partis qui entrent en compétition pour mettre en place les responsables communaux. Nous, en tant qu'association, nous ne participons pas, mais nos membres peuvent participer individuellement à cette compétition politique». Un membre d'une coopérative va dans le même sens : «S'ils [les élus] nous font appel, on assiste à des rencontres. Si c'est politique, on ne s'en mêle pas. S'il s'agit du bien-être de la commune, si nous sommes invités, nous donnons notre contribution ».

27 Par ailleurs, le fait que les acteurs de la société civile proclament leur apolitique illustre les contradictions des processus dites de démocratisation et de promotion de la citoyenneté. A ce propos, la plupart des acteurs de la société civile interrogés s'accordent à dire que l'introduction des principes démocratiques dans un contexte où l'organisation sociale et politique a fonctionné et fonctionne encore dans une certaine mesure selon un ordre très hiérarchisé, à l'instar de la société mossi (où il $\mathrm{y}$ a « un système de roi, de chefs et de sujets »), prendra du temps. Pour un responsable d'une association, " on peut voter ou battre des campagnes, mais il faut d'abord une éducation de la population pour que la démocratie vive réellement ". La problématique de l'éducation renvoie, entre autres, à celle de l'analphabétisme dont tout le monde reconnait l'acuité. En effet, la création de la mairie et l'urbanisation renforcent le recours à l'écrit, notamment dans les rapports entre les citoyens et les institutions (mairie, écoles, centres de santé, services déconcentrés, projets de développement). Or si les gens ne savent pas lire et écrire, il devient difficile, voire impossible, d'entreprendre certaines actions qui exigent la maîtrise de l'écriture. On pourrait même dire que tant que les populations ne seront pas suffisamment conscientisées que l'adaption de nouvelles pratiques peuvent améliorer leurs conditions de vie et compenser la diminution de la solidarité villageoise, elles continueront à bricoler pour survivre dans une sorte de jungle où les difficultés d'assurer la survie des siens sont grandes face à la détérioration de l'environnement social (émergence de l'individualisme et d'autres pratiques liées à l'urbanisation : prostitution, banditisme, etc.), économique (cherté de la vie, économie marchande, ...), et politique (néo-patrimonialisme, appât du gain, corruption, etc.).

28 Notre analyse met en évidence deux principaux enjeux de la décentralisation : promouvoir la participation de la population aux processus décisionnels et aux choix des priorités du développement communal d'une part, favoriser le dialogue et la communication entre les élus et la population d'autre part. Les propos d'un membre d'une association de jeunes, au chômage, sur l'impact de la création de la mairie, en témoignent : « Pour moi, la commune créée ou pas, il n'y a pas beaucoup de changements (janvier 2000). [...] Je dis qu'il n'y a pas eu de changements parce qu'on ne s'attaque pas à ce qui est primordial. Le problème de l'eau à Ziniaré est crucial. Si vous êtes ici en mai, vous allez voir vous-mêmes. Quand les gens parlent d'électricité ou de lotissements, moi je pense qu'il faut s'attaquer à ce qui est 
primordial. Même s'il y a quelques réalisations de forages, la population a aussi augmenté et augmente toujours. [...] Un autre problème c'est le chômage. On avait cru qu'avec les élections municipales (en 1995), il y aurait du travail pour beaucoup d'entre nous. Mais en réalité, il n'en est rien ». Ce témoignage illustre l'importance de la communication entre les élus et les habitants mais aussi la nécessité d'associer ces derniers dans le choix des actions prioritaires à réaliser. En effet, les besoins étant nombreux et les moyens de la mairie limités voire rares, les risques d'un désenchantement de la population sont nombreux si elle n'est pas suffisamment informée.

\section{La proximité des services de l'état civil : un acquis de la décentralisation}

Nos enquêtes montrent que malgré les difficultés de la mairie de Ziniaré à assumer effectivement l'ensemble de ses missions, plusieurs personnes interviewées apprécient positivement son avènement. En effet, la création de la mairie a plusieurs avantages, notamment la facilité et la rapidité des démarches administratives ainsi que la réalisation de certaines actions concrètes telles que la construction des infrastructures scolaires. Selon un enseignant, depuis la mise en place du conseil municipal élu, «Il y a des petits changements même si c'est en deça de ce qu'on attendait. Sur le plan administratif, il est possible d'avoir un papier à la mairie le même jour, ce qui n'était pas le cas auparavant. Maintenant le Maire est là ou s'il n'est pas là, il délègue toujours ses pouvoirs à un conseiller municipal qui peut signer un document en son absence. Or, avant, le préfet pouvait s'absenter pendant deux semaines et tout était bloqué jusqu'à son retour. Donc il faut reconnaître qu'il y a eu un changement. (...)».

\section{L'assainissement et la gestion des déchets : entre le monde rural et urbain}

A l'instar d'autres communes urbaines, les questions de l'assainissement et de la gestion des déchets sont à l'ordre du jour dans la commune de Ziniaré. En effet, l'habitat urbain, la densité de la population et les activités urbaines sont des facteurs qui exigent davantage une politique d'assainissement en milieu urbain qu'en milieu rural. Ces problématiques relèvent de plusieurs domaines de compétences que les lois sur la décentralisation attribuent à la mairie, notamment l'environnement et la gestion des ressources naturelles, l'eau, la santé et l'hygiène. Elles sont donc du ressort des politiques municipales.

31 Contrairement aux politiques foncières qui mobilisent plusieurs acteurs institutionnels à l'occasion des lotissements, le domaine de l'assainissement et de la gestion des déchets bénéficie de peu d'intérêt de la part des habitants de la commune. Pourtant son importance est indéniable, en particulier dans une perspective à moyen et à long termes. Comme gérer, c'est prévoir, l'analyse de l'évolution démographique de la population urbaine dans les pays ouest-africains entre 1960 et 1990 et les projections pour 2020 en témoignent. Si la proportion régionale d'urbains était de $40 \%$ de la population totale en 1990, elle devrait être de $63 \%$ en $2020^{7}$. Plus précisément, le Burkina Faso, qui comptait environ $25 \%$ de 
population urbaine en 1990, verra cette proportion doubler d'ici 2020. Autrement dit, si ces projections se confirment, la moitié de la population burkinabé habitera les agglomérations et les centres urbains d'ici huit ans. L'évolution démographique de Ziniaré, qui a quadruplé en quinze ans, passant de 3.451 habitants en 1985 à 13.803 habitants en 2000, semble confirmer ces tendances. Dès lors, les questions d'assainissement et de gestion des déchets constituent des enjeux importants. Elles pourraient constituer des créneaux dans lesquels les nouvelles autorités communales pourraient faire preuve de leur utilité; ce qui renforcerait leur légitimité.

32 Pour comprendre le peu d'intérêt accordé aux questions de l'assainissement et de la gestion des déchets dans les politiques communales, trois facteurs peuvent être avancés.

33 Le premier est lié à l'existence de plusieurs modes légitimes de justification des pratiques des acteurs. En effet, même si juridiquement ce sont les règles édictées par le conseil municipal qui priment, les observations sur le terrain montrent que les pratiques des acteurs suivent parfois d'autres logiques. François Wyngaerden ${ }^{8} \mathrm{a}$ bien montré la dualité de conceptions de l'utilité et l'utilisation des déchets ménagers dans les quartiers de Ziniaré. Pour les agriculteurs, les déchets ménagers sont conservés dans un tampuuré ${ }^{9}$ non loin de la cour en attendant la saison des pluies pour être déversés sur les champs afin de servir d'engrais. Pour d'autres catégories de la population, ceux qui souhaitent une ville plutôt moderne ainsi que les animateurs des services de la santé publique, les déchets doivent être enlevés et jetés à des endroits prévus à cet effet. De ce point de vue, c'est un problème de mentalités et de choix de modes de vie qui est en jeu ${ }^{10}$. Cela illustre comment un fait ou un événement devient ou ne devient pas un problème collectif voire public.

34 Le deuxième facteur est lié à l'absence de ressources humaines et financières au niveau de la mairie. Peu d'initiatives ont été prises dans ce domaine jusqu'en 2003. Selon les témoignages recueillis sur place, une commission a été mise en place pour réfléchir à cette question depuis le premier mandat du conseil municipal en 1995. En outre, une association locale a vu le jour en 2001 avec pour objectif de sensibiliser les habitants et d'initier, si possible, des actions de nettoyage et d'enlèvement des déchets. Si les réalisations de la mairie en matière d'entretien des principales voies de la commune et leur éclairage sont visibles, des défis restent à relever en matière de viabilisation des zones loties : construction des caniveaux, adduction d'eau, aménagement des sites pour les déchets, etc. Cette situation illustre le phénomène déjà mentionné du décalage entre les missions assignées à la mairie dans le développement communal et les moyens dont elle dispose. Autrement dit, le conseil municipal possède la légitimité de produire des règles et des normes sur son territoire mais elle ne dispose pas de ressources (au sens large du terme) nécessaires pour les faire respecter ${ }^{11}$. Le témoignage d'un habitant de Ziniaré à qui nous avons demandé si un citoyen peut interpeller les élus sur les questions de l'assainissement illustre ce qui précède: "Moi personnellement, je me suis déjà adressé directement au maire comme quoi nous avions un voisin qui cultivait dans sa parcelle et qu'au temps des moustiques, nous étions menacés (par le paludisme notamment). Le maire a réagi en envoyant quelqu'un qui n'a pas fait son travail. Les gens ont semé. Le maire a même fait passer un communiqué radiodiffusé pour interdire aux gens de semer dans les parcelles. » 
35 Cet exemple pose le problème de la mise en culture des jardins-cours des parcelles loties. Les cultures favorisent en effet la prolifération des moustiques et par là le paludisme. Contrairement au milieu rural où la coexistence entre les habitations et les cultures va de soi, il n'en est pas de même en ville. Ici, les exigences de la santé publique qui impliquent l'assainissement des lieux et celles de la production des biens pour certains habitants qui veulent maximiser l'exploitation de leur espace, s'avèrent contradictoires. D'où la nécessité d'une instance suffisamment légitime pour assurer la régulation sociale et politique, c'est-à-dire opérer des arbitrages, dans un environnement en voie d'urbanisation et qui est caractérisé par un pluralisme institutionnel et normatifs.

36 Le troisième facteur relève $d u$ fonctionnement et des mécanismes de la coopération internationale. La commune de Ziniaré, comme la plupart des petites communes urbaines d'ailleurs, dépend en grande partie de l'aide extérieure pour ses investissements. Or, l'assainissement et la gestion des déchets, en particulier dans les petites villes, ne figure pas parmi les domaines d'intervention prioritaires des bailleurs de fonds. Il en résulte, entre autres, une absence quasi totale d'actions de sensibilisation ou de réalisations significatives en la matière, que ce soit de la part de la mairie ou des associations et ONG locales, des projets de développement, voire même des services techniques de l'Etat. Autrement dit, l'agenda des bailleurs de fonds, bilatéraux ou multilatéraux, influe directement ou indirectement sur les politiques menées localement et sur les marges de manœuvre que peuvent avoir les autorités municipales. Face à l'Etat central qui a longtemps hésité à concrétiser la promesse de transférer de façon concomitante les compétences et les ressources au niveau local, les autorités communales comptent sur les donateurs pour tenir les promesses faites à leurs électeurs. Lorsque ceux-ci n'interviennent pas dans tel ou tel domaine tel que l'assainissement, celui-ci est laissé pour compte. Les autorités locales semblent ainsi avoir trop d'attentes envers les interventions extérieures et ne réalisent pas probablement que cela crée une dépendance. Or, les acteurs communaux en général et les autorités en particulier ne pourront assumer la gestion des affaires locales qu'à condition qu'ils apprennent à compter d'abord sur les ressources locales pour répondre aux besoins de leur milieu.

\section{Pluralisme institutionnel local et production normative}

37 Les recherches menées à Ziniaré mais aussi dans les cinq autres communes urbaines mettent en évidence la cohabitation de plusieurs institutions qui ont chacune ses sources de légitimités. Elles contribuent à produire des normes tout aussi légitimes en fonction des champs de compétences des unes et des autres. Si cette cohabitation ne pose pas beaucoup de problèmes, à ce stade du moins, c'est parce que ces acteurs institutionnels développement des relations de collaboration, de complémentarité et de concurrence de façon à garder l'équilibre dans les rapports de forces en présence.

38 Un examen de l'émergence de nouveaux phénomènes et les modalités de leur gestion au niveau communal, permet de mieux comprendre les processus de production des normes en cours. Prenons deux types de conflits : un conflit entre un commerçant - plus précisément un gestionnaire de télé-centre et un client qui 
refuse de payer ou bien qui n'as pas assez d'argent pour payer les unités utilisées ; et d'autre part, les conflits relatifs aux bagarres qui éclatent au marché. Selon les informations recueillies sur place, les conflits susceptibles de perturber l'ordre public relèvent des missions de la gendarmerie et de la police. L'exemple d'un gestionnaire de télé-centre illustre le rôle clé des forces de l'ordre pour la bonne marche des activités privées. Selon ses dires, "Quand les gens n'ont pas assez d'argent pour payer, on retire souvent leurs pièces d'identité pour qu'ils reviennent payer. (...) Quand ce sont les gens qui viennent de Ouagadougou ou de Kaya, c'est plus difficile avec eux. On appelle la police pour régler cela, parce que s'ils rentrent à Ouagadougou sans leurs pièces, ils peuvent avoir des problèmes. Mais si c'est quelqu'un qui vient de la province (d'Oubritenga), il peut laisser les pièces et revenir payer après. Quand on amène quelqu'un qui ne peut pas payer au commissariat, la police prend les coordonnées et la personne peut revenir payer plus tard. On a déjà amené deux personnes de la province d'Oubritenga qui voulaient faire la bagarre. Lorsqu'on demande à la personne de l'amener à la police et qu'il refuse, on appelle le 17 et la police vient ici ».

39 Ce témoignage illustre les nouveaux problèmes résultant de l'apparition de nouvelles activités et de nouveaux outils (les nouvelles technologies) dans cet espace en voie d'urbanisation. Il met également en évidence les modes de prise en charge de ces problèmes urbains par les institutions publiques contrairement à la campagne où ce sont surtout les institutions coutumières qui assurent la régulation sociale. Autrement dit, l'urbanité va de pair avec l'émergence de nouvelles normes (qui ne remplacent pas complètement les anciennes) régissant les relations entre les hommes entre eux, mais aussi entre ceux-ci et les institutions. Un témoignage sur la gestion des conflits au marché de dolo ${ }^{12}$ de Ziniaré tend à le confirmer : "Quand il y a des bagarres, la police vient et conduit les bagarreurs en prison jusqu'au lendemain. Comme ils ont bu, il faut attendre le lendemain pour trancher le conflit. Peu importe l'issue du conflit, ceux qui sont arrêtés payent la contravention». Dans certaines situations en effet, la punition est un moyen de responsabiliser les gens, de les socialiser sur l'importance du respect des règles de l'ordre public.

\section{Conclusion : quel rôle pour la socio-anthropologie dans la mise en place des institutions municipales?}

La compréhension des processus sociopolitiques dans lesquels les réformes institutionnelles ont lieu fait partie intégrante, formellement ou non, du processus de construction des institutions municipales. La démarche de recherche-action privilégiée par la Commission nationale de la décentralisation (CND) du Burkina Faso pour formuler, à l'attention du Gouvernement, un projet de politique de décentralisation en 1998 mais aussi lors de sa révision en 2001 en témoigne. En effet, la CND a eu recours à plusieurs études menées par des universitaires et experts nationaux et internationaux sur le foncier, les pouvoirs locaux, le rôle de la femme, le renforcement de la maîtrise d'ouvrage communale, le bilan des premières élections communales, etc. Les résultats de ces recherches ont alimenté le débat public à travers des réunions de sensibilisation sur les Textes de lois sur la décentralisation et contribué, modestement sans doute, à orienter les décisions 
politiques. En rendant compte les multiples dimensions et implications des réformes institutionnelles envisagées, ces études ont guidé d'une certaine les choix des politiques. Cependant, les analyses des sciences sociales constituent un des éléments parmi d'autres facteurs sur lesquels les politiques se basent dans leur prise de décision aussi bien sur les actions à mener que sur le calendrier de leur mise en œuvre.

41 Dans tous les cas, l'analyse des enjeux, des contraintes et des potentialités des politiques de construction des institutions municipales présentée ici constitue un exemple du rôle important qu'un socio-anthropologue peut jouer dans ce processus.

42 Plus globalement, un socio-anthropologue peut jouer un triple rôle dans le processus complexe de construction des institutions publiques locales ou nationales. Son premier rôle pourrait être de mobiliser les outils analytiques des disciplines des sciences sociales pour expliciter les processus sociaux, économiques, culturels et politiques des sociétés étudiées. Il n'est pas besoin de rappeler que comprendre, c'est déjà agir. Ensuite, son deuxième rôle serait de rendre compte des enjeux, des contraintes et des potentialités des actions à mener et des acteurs multiples qui y interviennent. Troisièmement enfin, il soumettrait les résultats de ses recherches aux décideurs et aux autres acteurs sociaux et participerait au débat public en tant que citoyen en vue des décisions éclairées. En effet, le socio-anthropologue professionnel est non seulement scientifique, mais aussi un citoyen qui ne peut pas se sentir indifférent de ce qui se passe dans sa communauté ou sa cité.

\section{BIBLIOGRAPHIE}

Bouju, J., 2002 (22-25 mai). «Espace public et citoyenneté à Bobo-Dioulasso : la salissure de la ville comme enjeu politique ». Communication au colloque « La gouvernance au quotidien en Afrique », APAD \& Centre d'études africaines, Leiden.

Commission Nationale De La Décentralisation (CND), 2002. Les Textes d'Orientation de la Décentralisation (TOD) du Burkina Faso modifiés, Ouagadougou, Ed. Shaifa.

Commission Nationale De La Décentralisation (CND), avril 1999. Les Textes d'Orientation de la Décentralisation (TOD) du Burkina Faso. Ouagadougou.

IDEA, janvier 1998. La Démocratie au Burkina Faso. Capacity - Building Series nº.

Hilgers, M., 2006. "Voter à Koudougou: la soumission d'une ville rebelle ? ", Politique africaine, n¹01: 42-62.

-. 2008. « Politiques urbaine, contestation et décentralisation. Lotissemement et représentations sociales au Burkina Faso », Autrepart, n47: 209-226.

-. 2009. Une ethnographie à l'échelle de la vielle. Urbanité, histoire et reconnaissance à Koudougou. Karthala, Paris. 
Jacob, J.-P., avril 2002. La tradition du pluralisme institutionnel dans les conflits fonciers entre autochtones. Le cas du Gwendégué (Centre Ouest Burkina Faso). Document de travail de l'UR 095, $n^{\circ}$ 3, IRD REFO.

Laurent, P.-J., 1995. Les pouvoirs politiques locaux et la décentralisation au Burkina Faso. Cahiers $d u$ CIDEP, n²6, Louvain-la-Neuve.

PDM et Club du Sahel (OCDE), 2001. Gérer l'économie localement en Afrique. Manuel ECOLOC. Tome 1. Paris.

Sebahara, P., juillet-août 1998. « Réflexions sur la société civile », Le Courrier ACP-UE, n¹70.

-. 2000. Acteurs et enjeux de la Décentralisation et du Développement local. Expériences d'une commune du Burkina Faso. Document de réflexion ECDPM n 21. Maastricht: ECDPM.

-. 2004. Suivi et évaluation des appuis à la décentralisation et à la gouvernance locale. Le cas du Burkina Faso. Document de réflexion ECDPM, n55. Maastricht: ECDPM.

Sebahara, P. ET Sawadogo R.A., 2004. « Historique de la décentralisation au Burkina Faso », in Laurent P.-J., Nyamba A., Dassetto F., Ouedraogo B. et Sebahara P. (eds), Décentralisation et citoyenneté au Burkina Faso. L'Harmattan et Academia Bruylant, Paris et Louvain-la-Neuve.

Sebahara, P. \& Sindogo F., 2004. « Décentralisation et citoyenneté communale. Réflexions sur la construction de l'espace public municipal ». in Laurent P.-J., et al. (eds), Décentralisation et citoyenneté au Burkina Faso. L'Harmattan et Academia Bruylant, Paris et Louvain-la-Neuve : 357-398.

Wyngaerden, F., « La gestion des déchets dans une ville émergente », in Laurent P.-J., Nyamba A., Dassetto F., Ouedraogo B.et Sebahara P. (dir.), Décentralisation et citoyenneté au Burkina Faso.

L'Harmattan et Academia Bruylant, Paris et Louvain-la-Neuve, 2004: 399-422.

\section{NOTES}

1. International IDEA, La Démocratie au Burkina Faso. Capacity - Building Series $n^{\circ} 4$, janvier 1998, p.52. Voir aussi Antoine R. Sawadogo et P.Sebahara, « Historique de la décentralisation au Burkina Faso », dans P.-J. Laurent, A. Nyamba, F.Dassetto, B.Ouedraogo et P.Sebahara (dir.), Décentralisation et citoyenneté au Burkina Faso. L'Harmattan et Academia Bruylant, Paris et Louvain-la-Neuve.

2. Commission Nationale de la Décentralisation (CND), Les Textes d'Orientation de la Décentralisation (TOD) du Burkina Faso modifiés, Ouagadougou, Ed. Shaifa, 2002, 125p.

3. CND, Les Textes d'Orientation de la Décentralisation (TOD) du Burkina Faso : Loi n041/98/AN, article 57.

4. Sur la gestion des affaires locales en milieu rural burkinabé, voir par exemple J.-P. Jacob, La tradition du pluralisme institutionnel dans les conflits fonciers entre autochtones. Le cas du Gwendégué (Centre Ouest Burkina Faso). Document de travail de l'UR 095, n 3, IRD REFO, avril 2002 ; P.-J.Laurent, Les pouvoirs politiques locaux et la décentralisation au Burkina Faso. Cahiers du CIDEP, n²6, Louvain-la-Neuve, 1995.

5. Les informations à la base de cette rubrique ont été recueillies par des interviews réalisées entre octobre 1999 et mars 2000. Les différents témoignages se réfèrent ainsi à l'expérience du premier conseil municipal.

6. «La société civile recouvre une pluralité d'acteurs, différents les uns que les autres aussi bien par leurs objectifs, leurs capacités organisationnelles, leur champ d'action, etc. En tout cas, les observateurs s'accordent à dire qu'elle est inséparable de l'Etat, avec lequel elle se construit en 
opposition dialectique», dans Pamphile Sebahara, "Réflexions sur la société civile », Le Courrier ACP-UE, n¹70, juillet - août 1998, p.95.

7. PDM et Club du Sahel (OCDE), Gérer l'économie localement en Afrique. Manuel ECOLOC. Tome 1. Paris, 2001, p.12.

8. F. Wyngaerden, «La gestion des déchets dans une ville émergente », dans P.-J. Laurent, A. Nyamba, F. Dassetto, B. Ouedraogo et P. Sebahara (dir.), Décentralisation et citoyenneté au Burkina Faso. L'Harmattan et Academia Bruylant, Paris et Louvain-la-Neuve, 2004, pp.399-422.

9. Tampuuré est le terme mooré qui signifie un tas d'ordures.

10. Voir Sebahara P. et Sindogo F., « Décentralisation et citoyenneté communale. Réflexions sur la construction de l'espace public municipal », dans P.-J. Laurent, et al. (dir), Décentralisation et citoyenneté au Burkina Faso. Op.cit., pp.357-398.

11. Une étude menée à Bobo-Dioulasso montre que les difficultés de la mairie à faire respecter ses règles de propreté publique contribue à sa perte de crédibilité vis-à-vis de la population et donc au processus de sa dé-légitimation. Voir J. Bouju, «Espace public et citoyenneté à BoboDioulasso: la salissure de la ville comme enjeu politique ». Communication au colloque «La gouvernance au quotidien en Afrique ", APAD \& Centre d'études africaines, Leiden, 22 - 25 mai 2002.

12. Le dolo est une bière locale fabriquée sur base de la farine de mil ou de sorgho.

\section{RÉSUMÉS}

L'article consiste en une analyse socio-anthropologique du processus de construction des institutions municipales au Burkina Faso, sur base des enquêtes menées principalement dans la commune de Ziniaré. Il met en évidence la perception, les enjeux, les contraintes et les potentialités de multiples acteurs qui y interviennent dans des rapports tantôt de négociation, de complémentarité ou de concurrence. L'analyse montre ainsi que la compréhension des processus sociopolitiques, culturels et économiques dans lesquels les réformes institutionnelles se mettent en place fait partie intégrante du processus de construction de ces institutions. Dès lors, le développement d'une socio-anthropologie de la construction institutionnelle susceptible d'aider à expliciter ces processus complexes, aussi bien au niveau local et national, et d'éclairer les acteurs sociaux est à la fois important et utile socialement.

The article consists of an anthropological analysis of the processes of the building of municipal institutions in Burkina Faso, on the basis of fieldwork in Ziniaré. It details the perception, the stakes, the constraints and the potentialities of multiple actors who intervene in these processes in relations of negotiation, complementarity and competition. The analysis demonstrates that the understanding of sociopolitical, cultural and economic processes in which institutional reforms are put into practice is part and parcel of the institution building process. Hence, the development of an anthropology of institution building that could help to make explicit these complex processes both at the local and national level and to enlighten social actors, is important and socially useful. 